\title{
Small Rho GTPase Rac1 determines human epidermal stem cell fate in vitro
}

\author{
LINLIN CHAI*, CHUAN CAO*, SHENG BI, XIA DAI, LU GAN, RUI GUO and SHIRONG LI \\ Department of Plastic and Reconstructive Surgery, Southwestern Hospital, \\ Third Military Medical University, Chongqing 400038, P.R. China
}

Received December 17, 2009; Accepted February 18, 2010

DOI: 10.3892/ijmm_00000397

\begin{abstract}
Epidermal stem cells (ESCs) are essential not only for tissue homeostasis but also for skin to respond to insults, but the mechanisms of stem cell regulation are unknown. To investigate the function of Rac1 in ESC development, we introduced either the dominant negative isoform or constitutively the active mutant of Rac1 in cultured human ESCs by using a retroviral vector and then analyzed the consequences. Upon activation, Rac1 increased surface $\alpha 6 / \beta 1$ integrin levels and promoted colony forming efficiency of ESCs. Conversely, dominant negative Rac1 caused a progressive reduction in growth rate, an inhibition of adhesiveness and a marked stimulation of terminal differentiation, without any effect on the cell cycle. These results were consistent with the role of Rac1 in determining the fate of ESCs by controlling their exit from the stem cell compartment. Our results reveal a novel biological role for Rac1 and provide new insights into the mechanism regulating ESCs.
\end{abstract}

\section{Introduction}

As the frontline barrier between body and environment, the epidermis is a multilayered structure consisting of rare but potent stem cells, short-lived progenitor cells termed transit amplifying cells (TA cells), and a large pool of differentiated cells derived from them. Among them, epidermal stem cells (ESCs) distinguish themselves with an unlimited self-renewal capacity and low probability of terminal differentiation. Stem cell characters make ESCs essential for tissue homeostasis as well as for the ability to respond to insults such as wound healing $(1,2)$. However, the underlying mechanisms of ESC

Correspondence to: Professor Shirong Li, Department of Plastic and Reconstructive Surgery, Southwest Hospital, Third Military Medical University, 29 Gaotanyan Main Street, Shapingba District, Chongqing 400038, P.R. China

E-mail: lishirong.cqzx@yahoo.com.cn

*Contributed equally

Key words: epidermal stem cells, Rho GTPases, Rac1, proliferation, differentiation, epidermis regulation are still unclear. Some studies established the importance of extracellular matrix adhesion, growth factors and cytokines in regulating ESC functions (3). Since Rac1 is a pleiotropic regulator of many cellular processes including integrin/growth factor signaling and cell-cell adhesion $(4,5)$, it might be crucial for ESC development.

Rac1 is a ubiquitously expressed member of the Rho family of small GTPases $(6,7)$. It cycles between an inactive GDPbound form and an active GTP-bound form. Conflicting results have been reported regarding the function of Rac1 in skin. The inducible deletion of Rac1 from the epidermis of adult mice in vivo results in ESC depletion (8). The conditional deletion of the Rac1 gene in mouse skin results in destruction of hair follicles (9). Expression of N17Rac1 in the epidermis of mice causes re-epithelialization in the protracted skin wound (10). Although these results suggest an important role of Rac1 in ESC, the underlying mechanism has not been addressed so far.

To study the relationship between Rac1 and ESC phenotypes, we induced either the dominant negative isoform or constitutively the active mutant of human Rac1 in ESC using a retroviral vector. Putative human ESCs were enriched based on collagen type IV adhesiveness and analyzed. Alterations on proliferation, differentiation, adhesiveness and colony forming efficiency were found in Rac1 mutant-expression ESCs. Together, these findings indicate that Rac1 helps determine the fate of ESC by regulating their exit from the stem cell compartment.

\section{Materials and methods}

Cell culture. Normal keratinocytes were isolated from human foreskins obtained during child circumcision. Our research was carried out following international and national regulations. Patients were informed of the ongoing research which was aimed at understanding the cellular and molecular mechanisms of ESCs. Accurate explanations and further information were also given about the forms to be filled and signed, in case of agreement to contribute to the research. Ethical approval was granted from the local ethics authority. All samples were processed according to the method of Rheinwald and Green (11). ESCs were separated according to their abilities to adhere to type IV collagen (Sigma). Briefly, primary epidermal cells from foreskins were plated onto culture flasks coated with type IV collagen $(20 \mu \mathrm{g} / \mathrm{ml})$ overnight at $4^{\circ} \mathrm{C}$, and incubated for $10 \mathrm{~min}$ at $37^{\circ} \mathrm{C}$. Non-adherent cells were rinsed off. Adherent cells were cultured in Defined Keratinocyte Serum-Free 
Medium (DK-SFM, Gibco) with medium changed every other day.

Production, concentration and transduction of recombinant lentivirus vectors. HIV-1-based lentivirus vector plasmids that express fusion protein of enhanced green fluorescent protein (EGFP) reporter gene and Rac1 mutants [dominant-negative mutant (N17Rac1, Rac1 DN), or constitutively active mutant (L61 Rac1, Rac1 CA)] were generous gifts from Dr Ying Wan (Department of Immunology, Third Military Medical University, China). FUGW was used as a control which expressed EGFP only. For preparation of pseudotyped lentivirus, packaging plasmid psPAX2 $(15 \mu \mathrm{g})$, envelope plasmid pMD2G $(5 \mu \mathrm{g})$, and vector plasmid DNA $(20 \mu \mathrm{g})$ were cotransfected into subconfluent 293FT cells with calciumphosphate precipitation (12). The viral supernatant was collected, filtered and concentrated by ultracentrifugation at $70,000 \mathrm{x}$ g for $2 \mathrm{~h}$ at $4^{\circ} \mathrm{C}$. For lentiviral transduction, ESCs were incubated with lentivirus at various 'multiplicity of infection' for $24 \mathrm{~h}$. Fusion protein expression was detected by fluorescent microscopy and flow cytometer on day 3 post-infection.

Growth and clonogenicity assays. Growth and clonogenicity assays were performed as described previously (13). For growth curves, 500 cells were plated per $35-\mathrm{mm}$ dish and cultured at $37^{\circ} \mathrm{C}$ in DK-SFM. Cells from triplicate dishes were harvested and counted every 2 days for up to 20 days. For clonogenicity assays, 500 ESCs were plated per $60-\mathrm{mm}$ Petri dish in triplicate. Cultures were grown for 14 days and stained with $0.1 \%$ crystal violet for $5 \mathrm{~min}$ at room temperature to visualize colony growth. All colonies containing $>40$ cells were scored in each dish, and colony-forming efficiency was calculated as percent of attached cells that formed colonies.

FACS. Infected ESCs were harvested, washed and fixed with $1 \%$ paraformaldehyde in PBS for $10 \mathrm{~min}$. For direct labeling, cells were incubated with specific primary antibody for $20 \mathrm{~min}$ in dark on ice. For indirect labeling, cells were incubated with primary antibody for $30 \mathrm{~min}$ at room temperature, washed twice, and then incubated with the second antibody for $20 \mathrm{~min}$ in dark on ice. For cell cycle analysis, cells were resuspended at $1 \times 10^{6}$ cells $/ \mathrm{ml}$ in PBS (containing $50 \mu \mathrm{g} / \mathrm{ml}$ propidium iodide and $0.1 \%$ Triton-X100) and incubated for $15 \mathrm{~min}$ on ice. Finally, cells were analyzed by a FACSAria flow cytometer (BD Biosciences) using the CellQuest software. The following antibodies were used, PE-conjugated anti-6 (BioLegend), anti-involucrin (Sigma), anti- $\$ 1$ and anti-mouse IgG TRITC conjugate (Santa Cruz).

Immunohistochemistry. ESCs were seeded on glass chamber slides coated with collagen IV overnight at $4^{\circ} \mathrm{C}$. When $60-70 \%$ confluence was reached, cells were washed, fixed and blocked in 2\% BSA in PBS. Cell surface integrin levels were detected by using PE-conjugated anti- $\alpha 6$ and FITC-conjugated antiCD71 antibodies (BioLegend).

Adhesion assays. ESCs ( $10^{3}$ per $35-\mathrm{mm}$ dish) were plated onto collagen I $(20 \mu \mathrm{g} / \mathrm{ml})$, collagen IV $(10 \mu \mathrm{g} / \mathrm{ml})$, or fibronectin $(10 \mu \mathrm{g} / \mathrm{ml})$ (all from Sigma) coated culture dishes in DK-SFM without Growth Supplement. Cells were allowed to adhere for
A

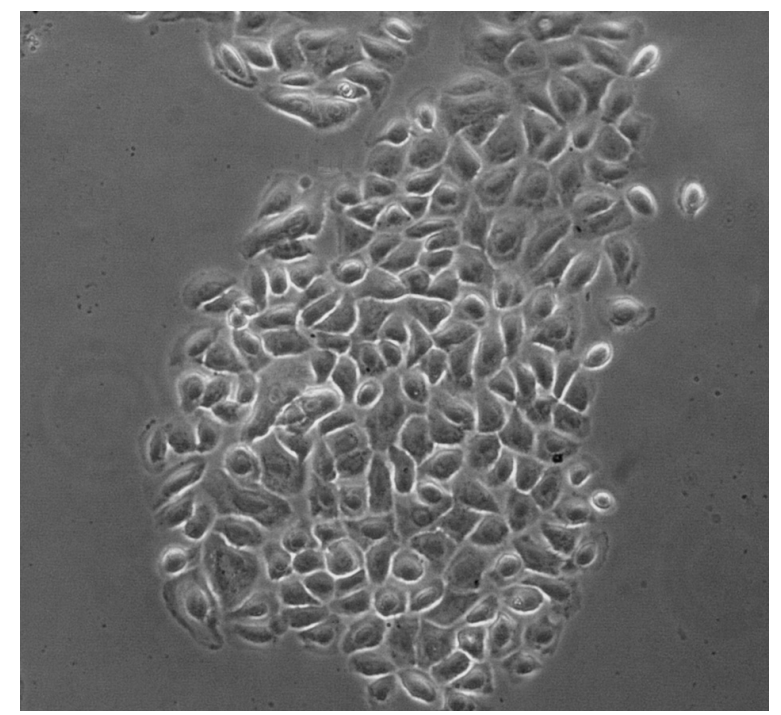

B
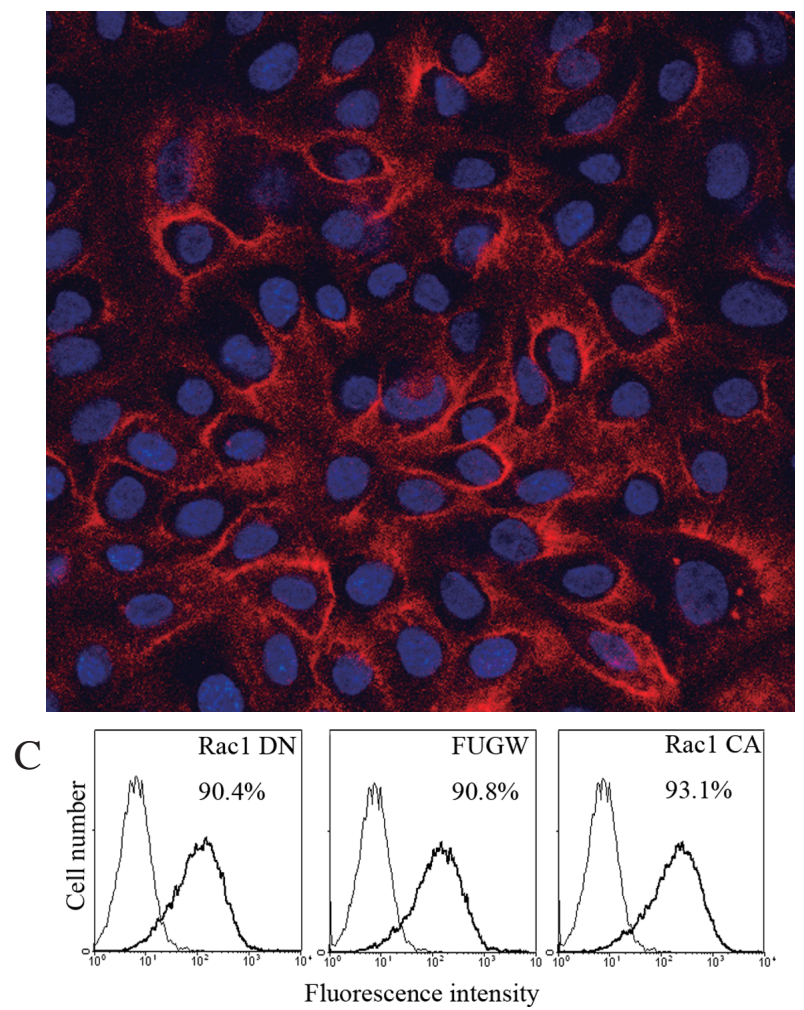

Figure 1. ESC enrichment and lentivirus infection. (A) Large colony formed by rapid adherent cell in culture. Epidermal cells were dissociated from human foreskin and allowed to adhere to collagen type IV-coated culture dishes for $10 \mathrm{~min}$. By 10 days culture in DK-SFM, these rapid adherent cells had formed large colonies. (B) Cell surface CD71 and $\alpha 6$ integrin expression. ESCs were seeded on glass chamber slides coated with collagen type IV. When 60-70\% confluent, cells were fixed in $4 \%$ paraformaldehyde and cell surface integrins were stained with PE-conjugated anti- $\alpha 6$ (red) and FITC-conjugated anti-CD71 antibodies (green). Almost all the cells from single colony were $\alpha 6^{\text {bri }}$ CD71 ${ }^{\text {dim }}$, indicating ESC phenotype. (C) Retrovirus infection rates. ESCs were incubated with lentivirus for $24 \mathrm{~h}$ and cultured in fresh medium for two more days. EGFP-positive cells were then measured by flow cytometer.

$1 \mathrm{~h}$, washed twice and then lysed with $0.9 \%$ Triton $\mathrm{X}-100$ in PBS. Adhesion was quantitated using a CytoTox 96 colorimetric kit (Promega) (14). 


\section{Results}

ESC enrichment and lentivirus infection. Stem cells are defined by their unique properties of slow-cycling nature and high proliferative potential. Basal keratinocytes with the cell surface phenotype $\alpha 6^{\text {bri }}$ CD71 ${ }^{\mathrm{dim}}$ exhibit many characteristics predicted of ESCs (15). Besides, high level $ß 1$ integrin expression in ESCs makes their enrichment easy by rapid adhesion to type IV collagen $(15,16)$. Therefore, based on collagen type IV adhesiveness, ESCs were enriched in our experiment. Approximately $50 \%$ of these rapidly adherent (RA) cells formed large colonies (Fig. 1A) and became confluent cultures within 2 weeks. We also examined the expressions of CD71 and $\alpha 6$ integrin by immunofluorescent staining to define RA cells. The result (Fig. 1B) showed that almost all cells from the single colony were $\alpha 6^{\text {bri }} \mathrm{CD} 71^{\mathrm{dim}}$. These results suggested that the RA population expanded and were used as ESCs later in the study.

The dominant-negative version of Rac1 (N17Rac1) and the constitutively active mutant of Rac1 (L61 Rac1) have been proved to be efficient tools in Rac1 functional studies (17). The lentivirus vector plasmids that express a fusion protein of EGFP and Rac1 mutants were verified by DNA sequencing (data not shown). After infection, EGFP could be detected by fluorescence microscope, and $>90 \%$ cells were positive analyzed by flow cytometer (Fig. 1C). These results indicated that high level expressions of Rac1 mutants in almost all ESCs could be achieved by lentivirus infection. Infected ESCs were then used for later experiments.

Effects of Racl on ESC proliferation and cell cycle. We next examined whether Rac1 had any effect on ESC proliferation. A growth curve was performed and is shown in Fig. 2A. Rac1 DN-expressing cells grew more slowly and reached saturation at a lower cell density than FUGW-expressing cells, while Rac1 CA-expressing cells grew more rapidly and reached a higher confluent density, indicating a positive correlation between Rac1 and ESC proliferation in this assay.

In order to investigate whether Rac1 controls the ESC proliferation by regulating the cell cycle, we stained cells from each population with propidium iodide, and determined DNA content by flow cytometry. However, the proportions of G1, $\mathrm{S}$, and $\mathrm{G} 2+\mathrm{M}$ cells were all statistically identical in Rac1 DN, FUGW, and Rac1 CA-expressing populations (Fig. 2B). There were no significant differences of cell cycle profile among the three groups.

Effects of Racl on ESC terminal differentiation. Given the lack of Rac1-induced cell cycle alteration, another explanation for the effects on ESC proliferation is that Rac1 promotes terminal differentiation. Therefore, expression of differentiation marker (involucrin) on Rac1 mutant-expressing ESCs was examined. The proportion of involucrin-positive cells was measured by flow cytometry (Fig. 2C). A 2-fold increase was detected in Rac1 DN-expressing cells (44.6\%) compared with control cells $(18.7 \%)$. There was no difference found between Rac1 CA-expressing and control cells. This suggests that downregulation of Rac1 stimulated ESC terminal differentiation.

Effects of Racl on ESC adhesion. ESCs locate in a specially arranged niche at the basal layer of the epidermis, which
A

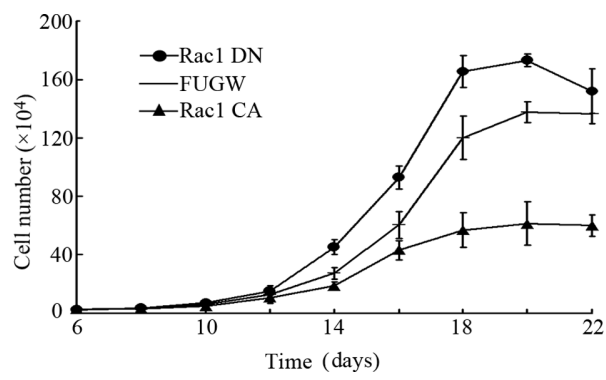

B

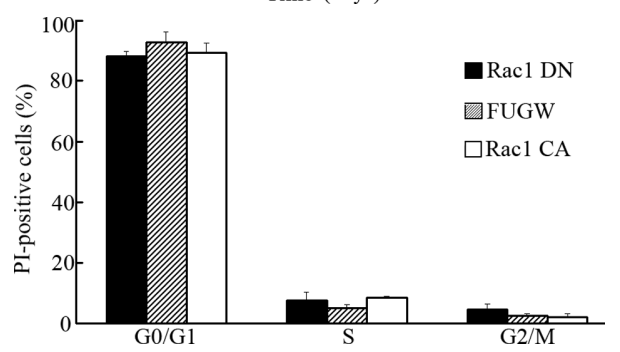

C

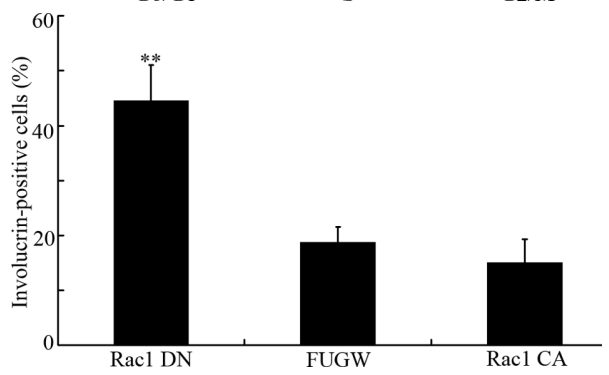

Figure 2. Effects of Rac1 on ESC proliferation, cell cycle and differentiation. (A) Effects of Rac1 on ESC proliferation. ESCs (500) expressing Rac1 DN, FUGW or Rac1 CA were plated per 35-mm dish and cultured in DK-SFM. Cells from triplicate dishes were harvested and counted every 2 days for up to 20 days. (B) Effects of Rac1 on ESC cell cycle. Cells were resuspended in PBS (containing $50 \mu \mathrm{g} / \mathrm{ml}$ propidium iodide and $0.1 \%$ triton-X100) and incubated for $15 \mathrm{~min}$ on ice, and then proportions of cells in different phases of the cell cycle were determined by flow cytometry. (C) Effects of Rac1 on ESC differentiation. Infected ESCs were harvested, washed and fixed in $1 \%$ paraformaldehyde in PBS for $10 \mathrm{~min}$. They were then sequently incubated with anti-involucrin antibody for $30 \mathrm{~min}$ at room temperature and TRITC conjugate anti-mouse IgG antibody for $20 \mathrm{~min}$ in dark on ice. The percentage of involucrin-positive cells was measured by flow cytometry. Data shown represent means \pm SEM values $(n=3) .{ }^{*} p<0.05,{ }^{* *} p<0.01$ as compared with FUGW-expressing cells. The results shown are representatives of three independent experiments.

provides protection for the long-lived stem cell population from harmful environmental mutagens and helps to maintain their stemness $(18,19)$. Migration into the suprabasal layer would drive entry of stem cell progeny into the transit amplifying cell compartment. Since our results suggested Rac1 DN expression induced ESC terminal differentiation without any effect on cell cycle, we wondered if Rac1 determined ESC fate by regulating the exit from the stem cell compartment. High level integrins on cell surface and adhesive ability of stem cells are actually necessary for maintenance of the stem cell compartment $(14,20)$. Therefore, the effects of Rac1 on ESC adhesion were examined.

For analysis of cell adhesion, equal numbers of cells were seeded for $1 \mathrm{~h}$ onto dishes coated with collagen I, collagen IV or fibronectin and the adherent cells were quantitated. Under all conditions, Rac1 DN-expressing cells showed a reduction of adhesion (Fig. 3A). Rac1 CA enhanced ESC adhesion on collagen I but not very efficiently. Notably, inhibition of 
A

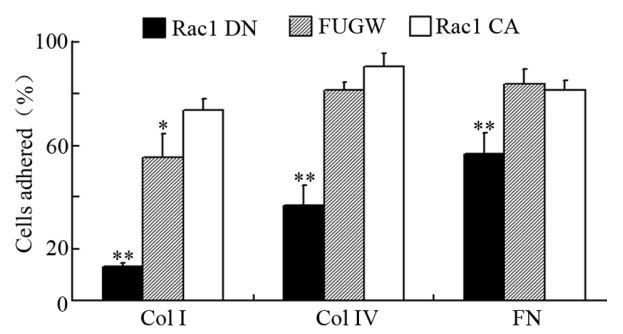

B

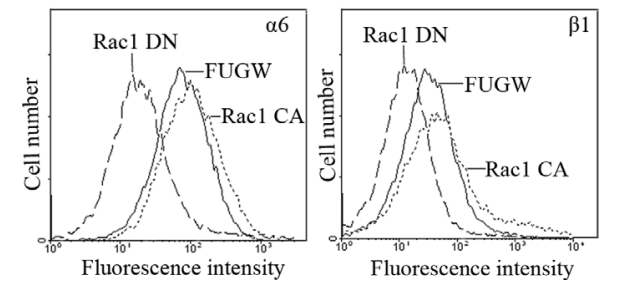

Figure 3. Effects of Rac1 on ESC adhesiveness. (A) Equal numbers $\left(10^{3}\right.$ per 35-mm dish) of ESCs expressing Rac1 DN, FUGW or Rac1 CA were seeded for $1 \mathrm{~h}$ into dishes coated with collagen I (Col I), collagen IV (Col IV) or fibronectin (FN). The number of adherent cells was determined by using a CytoTox 96 colorimetric kit. Data represent means \pm SEM values. ${ }^{*}<<0.05$, ${ }^{* *} \mathrm{p}<0.01$ as compared with FUGW-expressing cells. Each group consisted of 6 wells and similar results were obtained in each of the experiments. (B) Cell surface $\alpha 6$ and $\beta 1$ integrin levels were evaluated by flow cytometry. Marks on x-axis, maximum fluorescence (arbitrary units, log scale) of cells labeled with $\alpha 6-\mathrm{PE}$ or $\beta 1$-TRITC.

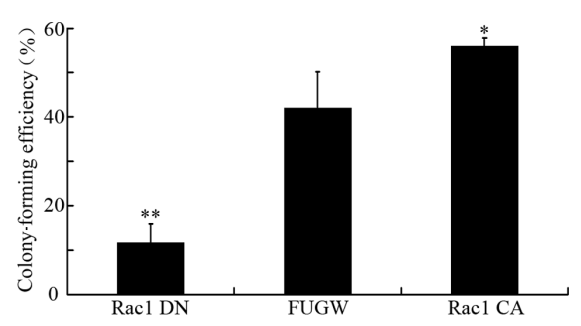

Figure 4. Effects of Rac1 on colony forming efficiency. Cells (500) in each group were plated on $20 \mu \mathrm{g} / \mathrm{ml}$ type IV collagen-coated $60-\mathrm{mm}$ tissue culture dishes, cultured for 14 days, and then stained with $0.1 \%$ crystal violet. All colonies containing $>40$ cells were scored on each dish, and colony forming efficiency was calculated as percent of attached cells that formed colonies. Data shown represent means \pm SEM values $(\mathrm{n}=3) .{ }^{*} \mathrm{p}<0.05$, ${ }^{* *} \mathrm{p}<0.01$ as compared with FUGW-expressing cells. The results shown are representatives of three independent experiments.

attachment was more pronounced when cells were plated on collagen I instead of collagen IV or fibronectin, indicating different roles of matrix in ESC development. Surface integrin level was also evaluated by flow cytometry (Fig. 3B). Both $\alpha 6$ and $\beta 1$ integrin significantly decrease in Rac1 DN-expressing populations, while there was no significant increase detected on Rac1 CA-expressing populations. The results suggest that Rac1 activity is required for matrix adhesiveness of ESCs especially on collagen I.

Effects of Racl on colony forming efficiency. Stem cells distinguish themselves from transit amplifying cells with an unlimited capacity of self-renewal. Therefore, clonogenicity is another valuable marker besides adhesiveness which could confirm the role of Rac1 in driving a transition from the stem to the transit amplifying compartment (13). Rac1 mutantsexpressing ESCs were seeded at clonal density and stained
14 days later to determine the total number of colonies formed. Only colonies that were rounded with $>40$ small basal cells were counted. As shown in Fig. 4, Rac1 DN caused a reduction of colony forming efficiency, while an increase was detected in Rac1 CA-expressing cells compared with control cells.

\section{Discussion}

The essential functions of the skin involve the homeostatic mechanisms of thermoregulation, maintenance of fluid balance, and protection against a variety of environmental insults. Both skin maintenance under steady-state conditions and tissue repair after injury are dependent on tissue-specific stem cells $(3,21,22)$. Stem cells may remain quiescent or proliferate under different circumstances. How are these decisions regulated is still unclear. Recently, research on Rac1 and keratinocytes has been carried out. Although the importance of Rac1 in ESC development had been pointed out, the results remain contradictory. The heterogeneity between stem cells and transit amplifying cells also hamper further investigation. In our experiment, ESCs were enriched by rapid adhesion to collagen IV. Significant alterations of both differentiation and adhesion were found in Rac1 mutant-expressing ESCs. Along with the change detected in the clonogenicity assay, all these results suggest that Rac1 helps determine the fate of ESCs by regulating their exit from the stem cell compartment.

Rac1 inhibition in ESCs suppressed adhesiveness, reduced colony forming, stimulated terminal differentiation, therefore promoted transition from stem cell to the transit amplifying cell compartment. There was no change found in the cell cycle. Proliferation reduction of Rac1 DN-expressing ESCs was attributed to fewer colonies formed and more differentiated cells produced. Similarly, a rapid depletion of stem cells after deletion of Rac1 from adult mouse epidermis was reported (8). Rac1 could impede the expression of premature differentiation markers in growing human keratinocytes, probably by exerting positive control on c-myc activity and its binding to specific promoters (23). Collectively the above indicate an important role of Rac1 in regulating ESC development. However, no alteration on either proliferation or differentiation was found by Chrostek et al in the epidermis of Rac1-deficient mouse mutants (9). Heterogeneity of interfollicular and follicular stem might be responsible for this discrepancy (see Discussion).

Whether constitutive activation of Rac1 has any effect on ESCs was also addressed in our experiment. Previously reported, overexpression of activated Rac1 in human keratinocytes blocked Myc-induced terminal differentiation and prevented the decrease in clonal growth that occurs on Myc activation $(13,24)$. In our study, other than the proliferation promotion, the increasing levels of $\alpha 6 / \beta 1$ integrins and the enhancement of colony forming efficiency were barely detected. This lack of alteration after Rac1 upregulation might be because the cells were already in an undifferentiated state. Rac1 may normally act as a steady factor and play a more important role during stress such as injury. Impaired epidermal wound healing in vivo upon inhibition or deletion of Rac1 was reported recently (10). However, excessively increased expression of Rac1 may relate to tumor development (5). 
Basal cells of the interfollicular epidermis (IFE) are continuous with those of the hair follicle and sebaceous gland, which form the specialized cells of the hair shaft and sebocytes. Whether the IFE, the hair follicle, and the sebaceous gland each have their own stem cell compartment, or whether there are single pluripotential stem cells that select a differentiation pathway depending on their microenvironment is unclear $(3,21,25)$. It was reported that follicular stem cells could be distinguished from interfollicular stem cells by their requirement of Rac1 (26). Constitutive, epidermis-specific deletion of Rac1 during murine embryonic development resulted in destruction of hair follicles but no major alterations in the interfollicular skin (9). In contrast to their conclusions, our results imply a regulating effect on interfollicular stem cells of Rac1, since ESCs isolated from human foreskins in our experiment are mostly classified as interfollicular stem cells $(27,28)$. A likely cause for this discrepancy is the different genetic background. It might be, however, that deletion of the Rac1 gene during embryonic development allows for a compensatory response that cannot be initiated in adult tissue. Alternatively, an alteration in the interfollicular skin might happen later and be less severe than hair follicular destruction, because hair follicle keratinocytes can repopulate the inter-follicular epidermis so that the skin intactness is kept $(1,29)$. Any confirmative reason needs further investigation.

The extent to which stem cell behavior is preprogrammed or environmentally regulated has long been a subject for debate. The extensive self-renewal capacity of the epidermis can be attributed not only to intrinsic regulators expressed within ESC, but also to microenvironmental or extrinsic factors produced by cells in the neighboring dermis $(3,21)$. As a pleiotropic regulator of many cellular processes, including integrin and growth factor signaling and cell-cell adhesion $(4,5)$, Rac1 could be a critical molecular regulator at the crossroad of the two compartments. In this context, our investigation supports that Rac1 determines the ESC fate in vitro by controlling their adhesiveness and terminal differentiation. We also provide the first evidence that the interfollicular stem cells can be regulated as well as follicular stem cells. These finding contribute to our understanding of basic mechanisms regarding stem cell development and may have future therapeutic implications for tissue engineering or disease treatment.

\section{Acknowledgements}

This work was supported in part by a grant from the National Natural Science Foundation of China (no.30600652).

\section{References}

1. Taylor G, Lehrer MS, Jensen PJ, Sun TT and Lavker RM: Involvement of follicular stem cells in forming not only the follicle but also the epidermis. Cell 102: 451-461, 2000.

2. Ito M, Liu Y, Yang Z, Nguyen J, Liang F, Morris RJ and Cotsarelis G: Stem cells in the hair follicle bulge contribute to wound repair but not to homeostasis of the epidermis. Nat Med 11: 1351-1354, 2005.

3. Janes SM, Lowell S and Hutter C: Epidermal stem cells. J Pathol 197: 479-491, 2002.
4. Etienne-Manneville S and Hall A: Rho GTPases in cell biology. Nature 420: 629-635, 2002.

5. Benitah SA, Valeron PF, van Aelst L, Marshall CJ and Lacal JC: Rho GTPases in human cancer: an unresolved link to upstream and downstream transcriptional regulation. Biochim Biophys Acta 1705: 121-132, 2004

6. Jaffe AB and Hall A: Rho GTPases: biochemistry and biology. Annu Rev Cell Dev 21: 247-269, 2005.

7. Wennerberg K and Der CJ: Rho-family GTPases: it's not only Rac and Rho (and I like it). J Cell Sci 117: 1301-1312, 2004.

8. Benitah SA, Frye M, Glogauer M and Watt FM: Stem cell depletion through epidermal deletion of Rac1. Science 309: 933-935, 2005.

9. Chrostek A, Wu X, Quondamatteo F, et al: Rac1 is crucial for hair follicle integrity but is not essential for maintenance of the epidermis. Mol Cell Biol 26: 6957-6970, 2006.

10. Tscharntke M, Pofahl R, Chrostek-Grashoff A, et al: Impaired epidermal wound healing in vivo upon inhibition or deletion of Rac1. J Cell Sci 120: 1480-1490, 2007.

11. Rheinwald JG and Green H: Serial cultivation of strains of human epidermal keratinocytes: the formation of keratinizing colonies from single cells. Cell 6: 331-343, 1975.

12. Kutner RH, Zhang XY and Reiser J: Production, concentration and titration of pseudotyped HIV-1-based lentiviral vectors. Nat Protoc 4: 495-505, 2009

13. Gandarillas A and Watt FM: c-Myc promotes differentiation of human epidermal stem cells. Genes Dev 11: 2869-2882, 1997.

14. Zhu AJ, Haase I and Watt FM: Signaling via beta1 integrins and mitogen-activated protein kinase determines human epidermal stem cell fate in vitro. Proc Natl Acad Sci USA 96: 6728-6733, 1999.

15. Tani H, Morris RJ and Kaur P: Enrichment for murine keratinocyte stem cells based on cell surface phenotype. Proc Natl Acad Sci USA 97: 10960-10965, 2000.

16. Jones PH and Watt FM: Separation of human epidermal stem cells from transit amplifying cells on the basis of differences in integrin function and expression. Cell 73: 713-724, 1993.

17. Mettouchi A, Klein S, Guo W, Lopez-Lago M, Lemichez E, Westwick JK and Giancotti FG: Integrin-specific activation of Rac controls progression through the G(1) phase of the cell cycle. Mol Cell 8: 115-127, 2001.

18. Tumbar T, Guasch G, Greco V, Blanpain C, Lowry WE, Rendl M and Fuchs E: Defining the epithelial stem cell niche in skin. Science 303: 359-363, 2004 .

19. Lavker RM and Sun TT: Epidermal stem cells: properties, markers, and location. Proc Natl Acad Sci USA 97: 13473-13475, 2000.

20. Kaur P and Li A: Adhesive properties of human basal epidermal cells: an analysis of keratinocyte stem cells, transit amplifying cells, and postmitotic differentiating cells. J Invest Dermatol 114: 413-420, 2000.

21. Kaur P: Interfollicular epidermal stem cells: identification, challenges, potential. J Invest Dermatol 126: 1450-1458, 2006.

22. Roh C and Lyle S: Cutaneous stem cells and wound healing. Pediatr Res 59: R100-R103, 2006.

23. Nikolova E, Mitev V, Minner F, Deroanne CF and Poumay Y: The inhibition of the expression of the small Rho GTPase Rac1 induces differentiation with no effect on cell proliferation in growing human adult keratinocytes. J Cell Biochem 103: 857-864, 2008.

24. Frye M, Gardner C, Li ER, Arnold I and Watt FM: Evidence that Myc activation depletes the epidermal stem cell compartment by modulating adhesive interactions with the local microenvironment. Development 130: 2793-2808, 2003.

25. Watt FM and Hogan BL: Out of Eden: stem cells and their niches Science 287: 1427-1430, 2000.

26. Castilho RM, Squarize CH, Patel V, Millar SE, Zheng Y, Molinolo A and Gutkind JS: Requirement of Rac1 distinguishes follicular from interfollicular epithelial stem cells. Oncogene 26: 5078-5085, 2007

27. Jones PH, Harper S and Watt FM: Stem cell patterning and fate in human epidermis. Cell 80: 83-93, 1995.

28. Legg J, Jensen UB, Broad S, Leigh I and Watt FM: Role of melanoma chondroitin sulphate proteoglycan in patterning stem cells in human interfollicular epidermis. Development 130: 6049-6063, 2003

29. Bereiter-Hahn J: Epidermal cell migration and wound repair. In: Biology of the Integument vol 2. Vertebrates. Springer-Verlag, Berlin, pp443-471, 1986. 